\title{
Concentrations of Radionuclides in Terrestrial Vegetation on the Hanford Site of Potential Interest to Native Americans
}

T. M. Poston

March 1995

Prepared for the U.S. Department of Energy under Contract DE-AC06-76RLO 1830

Pacific Northwest Laboratory

Operated for the U.S. Department of Energy

by Battelle Memorial Institute 


\section{DISCLAIMER}

This report was prepared as an account of work sponsored by an agency of the United States Government. Neither the United States Government nor any agency thereof, nor Battelle Memorial Institute, nor any of their employees, makes any warranty, expressed or implied, or assumesany legal liability or responsibility for theaccuracy, completeness, or usefulness of any information, apparatus, product, or process disclosed, or representsthat its use would not infringe privately owned rights. Reference herein to any specific commercial product, process, or serviceby trade name, trademark, manufacturer, or otherwisedoes not necessarily constitute or imply its endorsement, recommendation, or favoring by the United States Government or any agency thereof, or Battelle Memorial Institute. The views and opinions of authors expressed herein do not necessarily state or reflect those of the United States Government or any agency thereof.

\section{PACIFIC NORTHWEST LABORATORY operated by BATTELLEMEMORIAL INSTITUTE for the UNITED STATES DEPARTMENT OF ENERGY under Contract DE-AC06-76RLO 1830}

Printed in the United States of America

Available to DOE and DOE contractors from the

Office of Scientific and Technical Information, P.O. Box 62, Oak Ridge, TN 37831; prices available from (615) 576-8401. FTS 626-8401.

Available to the public from the National Technical Information Service, U.S. Department of Commerce, 5285 Port Royal Rd., Springfield, VA 22161. 
PNL-10397

UC-602

Concentrations of Radionuclides in Terrestrial Vegetation on the Hanford Site of Potential Interest to Native Americans

T. M. Poston

March 1995

Prepared for

the U.S. Department of Energy

under Contract DE-AC06-76RLO 1830

Pacific Northwest Laboratory

Richland, Washington 99352 


\section{SUMMARY}

Concentrations of ${ }^{90} \mathrm{Sr}$ and ${ }^{137} \mathrm{Cs}$ in Carey's balsamroot (Balsamorhiza careyana) and Gray's desertparsley (Lomatium grayi) were similar to concentrations observed in other plants collected on the Hanford Site and from offsite locations surrounding the Site as part of annual 'Hanford Site surveillance. Observed concentrations may be attributed to historic fallout more than to Hanford Site emissions,

although the observation that 200 Area plants had slightly higher concentrations of ${ }^{137}$ Cs than 100 Area plants is consistent with other monitoring data of radioactivity in soil and vegetation collected onsite. The present concentrations of ${ }^{90} \mathrm{Sr}$ and ${ }^{137} \mathrm{Cs}$ in balsamroot and parsley fluctuate around background levels with some of the higher observed concentrations of ${ }^{90} \mathrm{Sr}$ found on the Fitzner/Eberhardt Arid Lands Ecology (ALE) Reserve. Analytical results and summary statistics by species and location are presented in the appendixes. 


\section{ACKNOWLEDGMENTS}

The author expresses his appreciation to R. L. Dirkes, K. R. Price, P. R. Nickens, and associated staff of the Cultural Resources Group for critical reviews of this report. J. Dahl provided the initial screening and selection of plants and W. H. Rickard provided further consultation on sample selection and participated in sample collection with Radiation Protection Technologists. M. K. DeSmet provided editorial and publication assistance. 


\section{CONTENTS}

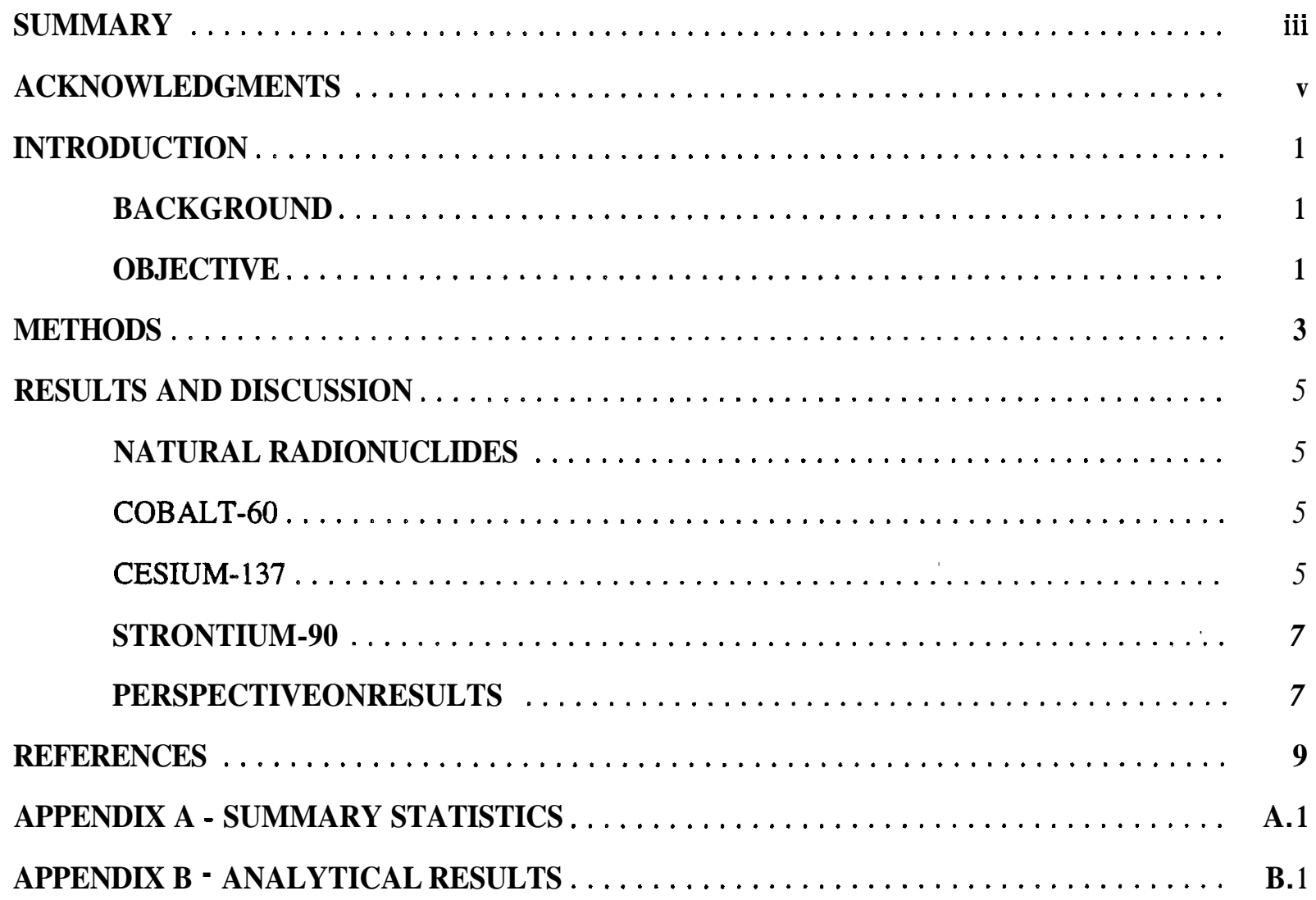




\section{FIGURES}

1 Sampling Locations of Balsamroot and Parsley. April and May. $1994 \ldots \ldots \ldots \ldots \ldots$. . 4

2 Box Plot of ${ }^{137} \mathrm{Cs}$ in Terrestrial Vegetation $\ldots \ldots \ldots \ldots \ldots \ldots \ldots \ldots \ldots \ldots \ldots$

3 Scattergram of ${ }^{137} \mathrm{Cs}$ in Terrestrial Vegetation $\ldots \ldots \ldots \ldots \ldots \ldots \ldots$

4 Box Plot of ${ }^{90} \mathrm{Sr}$ in Terrestrial Vegetation $\ldots \ldots \ldots \ldots \ldots \ldots \ldots \ldots \ldots \ldots$

5 Scattergram of ${ }^{90} \mathrm{Sr}$ in Terrestrial Vegetation $\ldots \ldots \ldots \ldots \ldots \ldots \ldots \ldots \ldots$

\section{TABLE}

1 Terrestrial Plants on the Hanford Site of Potential Interest to Native Americans $\ldots . \ldots \ldots \quad 2$ 


\section{INTRODUCTION}

The U.S. Department of Energy's (DOE's) Hanford Site was established in 1943 in southeastern Washington State for nuclear materials production. Although the Site is quite large $\left(1450 \mathrm{~km}^{2}\left[560 \mathrm{mi}^{2}\right]\right)$, only about $6 \%$ of the Site has been developed for industrial purposes (Woodruff et al. 1993). For the Site's first 45 years of existence, its principal mission was the production of plutonium for national defense and the management of resulting wastes. The Hanford Site is currently being remediated and cleaned up under the direction of the Hanford Federal Facility Agreement and Consent Order (1989 as amended), commonly referred to as the Tri-Party Agreement. As cleanup proceeds, more public access will be allowed onsite. The interest and potential-use of the Site by regional Native American tribes is great, and their anticipated access to the Site warrants additional investigations.

\section{BACKGROUND}

Native Americans use many wild plants, some of which are found on the Hanford Site. They were used for food as well as for medicinal and religious practices. These species and their uses have been briefly summarized by Sackschewsky et al. (1992). This group of plants was reviewed with Hanford Site scientists and a staff botanist for the Yakama Indian Nation and pared down to eight species for sampling consideration (Table 1). Further review with Site botanists identified two plants most suitable for sampling because they were relatively large plants and were common on the Site; Carey's balsamroot (Balsamorhiza careyana) and two parsley species: Gray's desertparsley (Lomatium grayi) and turpentine springparsley (Cymopteris terebinthinus). The roots of parsley are very small and they were not sampled. While other species could have been sampled, for this preliminary sampling effort, this study focused on these species as generally representative of other terrestrial vegetation.

Additional information is available on radionuclide concentrations in other endemic Site vegetation in annual Site environmental monitoring reports produced by the Pacific Northwest Laboratory. Routine surveillance of Site vegetation has provided data on radionuclide concentrations in mixed samples of big sagebrush (Artemisia tridentata) and rabbitbrush (Chrysothamnus sp.). Additionally, an extensive evaluation of radionuclide contamination in riparian vegetation was conducted from 1990 through 1992 (Antonio et al. 1993).

\section{OBJECTIVE}

The objective of this study was to obtain and report data on the concentrations of radionuclides in endemic plants on the Hanford Site that may be of interest to regional tribes, particularly species that have not normally been sampled. In April and May, 1994, two species of parsley and one species of balsamroot were collected from three distinct areas on the Hanford Site for analysis of gamma emitting radionuclides and ${ }^{90} \mathrm{Sr}$. 
Table 1. Terrestrial Plants on the Hanford Site of Potential Interest to Native Americans(a)

Plant

\{Common Name)

Allium macrum

(rock onion)

$\begin{array}{ccc}\begin{array}{c}\text { Amelanchier } \\ \text { alnifolia }\end{array} & \text { fruit } & \text { autumn } \\ \text { (western } & & \\ \text { serviceberry) } & & \\ & & \\ \text { Balsamorhiza } & \text { stalk } & \text { spring } \\ \begin{array}{c}\text { careyana } \\ \text { (Carey's } \\ \text { balsamroot) }\end{array} & \text { root } & \\ & & \end{array}$

Lewisia rediviva bulb

(bitterroot)

Lomatium grayi $\quad$ root $\quad$ spring
$\begin{aligned} & \text { (Gray's } \\ & \text { desertparsley) }\end{aligned}$

$\begin{array}{ccc}\text { Perideridia } & \text { root } & \text { spring } \\ \text { gairdneri } & & \\ \text { (Gairdner's } & & \\ \text { yampah) } & & \end{array}$

\section{Prunus}

virginiana

(chokecherry)

Harvest

Date

spring

spring
Local on rocky slopes Umtanum/Rattlesnake Ridges Artemisia rigida-Poa, Intermediates, Eriogonum thymoides-Poa, Eriogonum douglasii-Poa

Common \& widespread; rocky-silty soils along drainages, Rattlesnake Mountain, Snively Canyon Artemisia rigida-Poa

Eriogonum thymoides-Poa

White Bluffs \& Rattlesnake Hills

Symphoricarpus phase of Festuca-Symphoricarpus, Artemisia tripartita-Agropyron spicatum (might not be common)

summer autumn

autumn
Widespread in moist areas, e.g., Snively Canyon, Hanford Townsite

Festuca-idahoensis-Symphoricarpus, Purshia tridentata-Festuca idahoensis Crataegus douglasii-Heracleum lanatum

Local in drainages of Rattlesnake Ridge Crataegus douglasii-Heracleum lanatum

(a) See Downs et al. (1993) and Daubenmire (1970) for more detailed descriptions of plant associations and associated soil types. 


\section{METHODS}

The species collected were Carey's balsamroot and two species of parsley: Gray's desertparsley and turpentine springparsley. These species were chosen over other species of interest to Native Americans because of their great abundance on the Site. Balsamroot samples consisted of roots, or leaves and stems. Parsley samples consisted of leaves and stems only; the roots were too small to obtain sufficient sample mass for analysis. Each sample was a composite of multiple plants collected at each location. Samples were submitted to International Technology Laboratories (ITAS), Richland, Washington, for drying and radionuclide analysis. All results are reported as pCi/g dry weight. Results are reported for ${ }^{7} \mathrm{Be},{ }^{40} \mathrm{~K},{ }^{60} \mathrm{Co},{ }^{137} \mathrm{Cs}$, and ${ }^{90} \mathrm{Sr}$.

Samples where collected from three general areas. The Fitzner-Eberhardt Arid Lands Ecology Reserve (ALE) along the 1200 Foot Road was sampled as an area generally upwind from the industrialized areas. Plants growing on land adjacent to two industrialized areas were also sampled; the 200 Area plateau in areas to the south and east of the 200-W and 200-E Areas, and the 100 Areas extending from east of 100-N to 100-F (Figure 1). The 200 Areas contain shutdown facilities that have processed irradiated fuel elements from the production reactors and currently store radioactive wastes. The 100 Areas include a series of 7 distinct areas located along the Columbia River where shutdown nuclear production reactors are located.

Data are graphically presented as scattergrams showing all data and box plots. The box plot shows the median, the 25th percentile (bottom of box), and 75th percentile (top of box) concentrations. Box figures with additional bars show the 10th and 90th percentiles and symbols above and below those bars show individual results outside-of the 10th and 90th percentile concentrations when $\mathrm{N} \geq 4$. Because of the limited number of samples collected, the box plots are of marginal value for depicting the distribution of the data, however it is assumed that the data follow a log-normal distribution and the median concentration provides a better estimate of central tendency than the mean. Summary statistics of radionuclide concentrations by location and individual analytical results for each species, sample portion, and location are found in the appendixes. 


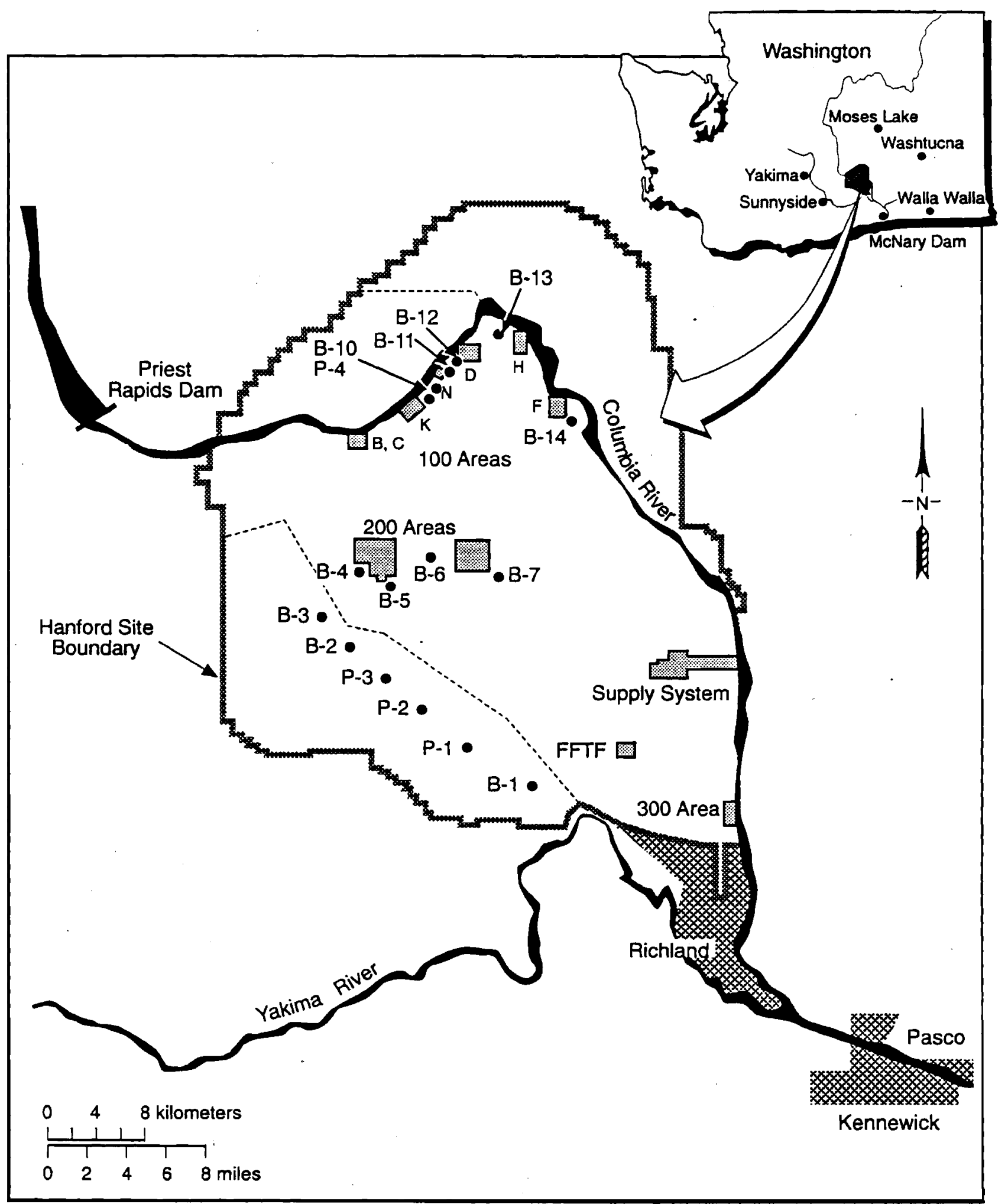

S9410026.1

Figure 1. Sampling Locations of Balsamroot (B) and Parsley (P), April and May, 1994 


\section{RESULTS AND DISCUSSION}

Both naturally occurring and manmade radionuclides were found in plant samples collected during this study. Natural radionuclides found in plant samples were ${ }^{7} \mathrm{Be}$ and ${ }^{40} \mathrm{~K}$; manmade radionuclides were ${ }^{60} \mathrm{Co},{ }^{137} \mathrm{Cs}$, and ${ }^{90} \mathrm{Sr}$.

\section{NATURAL RADIONUCLIDES}

Potassium-40 and ${ }^{7} \mathrm{Be}$ were routinely measured in plant samples. Both of these radionuclides occur naturally and their presence is not unexpected. Beryllium-7 is of atmospheric origin and has a relatively short half-life (53.6 d). It was found in leaves, but not the roots of plants indicating lack of translocation to roots from the leaves and little indication of uptake from soil. Potassium- 40 was also found in higher concentrations in leaves than in roots. Median concentrations of ${ }^{40} \mathrm{~K}$ ranged from 31 to $42 \mathrm{pCi} / \mathrm{g}$ in leaves and 5.9 to $8.2 \mathrm{pCi} / \mathrm{g}$ in roots. Concentrations of $\mathrm{K}$ vary between different species of plants and by season (Rickard and Vaughan 1988).

\section{COBALT-60}

The analyses in general show that ${ }^{60} \mathrm{Co}$ was not found in plants collected from the 200 Area plateau or ALE, however 3 balsamroot samples collected from the 100 Areas had detectable amounts in either the roots or leaves. These concentrations of ${ }^{60} \mathrm{Co}$ were near the detection limit of $0.02 \mathrm{pCi} / \mathrm{g}$.

\section{CESIUM-137}

Cesium-137 was found only in balsarnroot, and almost exclusively in the roots from all areas sampled (Figures 2 and 3). Two of fourteen balsarnroot leaf samples contained ${ }^{137} \mathrm{Cs}$ at concentrations close to the detection limit of $0.02 \mathrm{pCi} / \mathrm{g}$. The concentrations of ${ }^{137} \mathrm{Cs}$ in balsamroot roots collected from the 200 Areas were greater than concentrations observed in samples collected at ALE. This observation is consistent with observations of the distribution of ${ }^{137} \mathrm{Cs}$ in soil and desert vegetation samples (consisting of rabbitbrush and big sagebrush) collected onsite during routine surveillance and reported in annual Site surveillance reports. The maximum and median onsite concentration of ${ }^{137} \mathrm{Cs}$ in balsamroot leaves collected from the 200 Areas was $0.05( \pm 0.0220$ counting error) and $0.02 \mathrm{pCi} / \mathrm{g}$, respectively. The maximum and median onsite concentration (sampled outside of nuclear facilities boundaries) of ${ }^{137} \mathrm{Cs}$ in routinely monitored desert vegetation sampled onsite from 1985 through 1993 was $0.36( \pm 0.04) \mathrm{pCi} / \mathrm{g}$ in the 200 Areas and $0.03 \mathrm{pCi} / \mathrm{g}$ for 92 samples, respectively. The median concentrations provide a better basis for comparison. The maximum and median concentration of ${ }^{137} \mathrm{Cs}$ collected at distant locations was $0.98( \pm 0.05) \mathrm{pCi} / \mathrm{g}$ (at Washtucna), and $0.02 \mathrm{pCi} / \mathrm{g}$ for the 37 samples collected at Washtucna, Moses Lake, Walla Walla, McNary Dam, Sunnyside, and Yakima, respectively. Collectively, these results show that ${ }^{137} \mathrm{Cs}$ in the species collected do not indicate any mechanism of elevated accumulation or bioconcentration relative to other desert vegetation that have been historically monitored. 


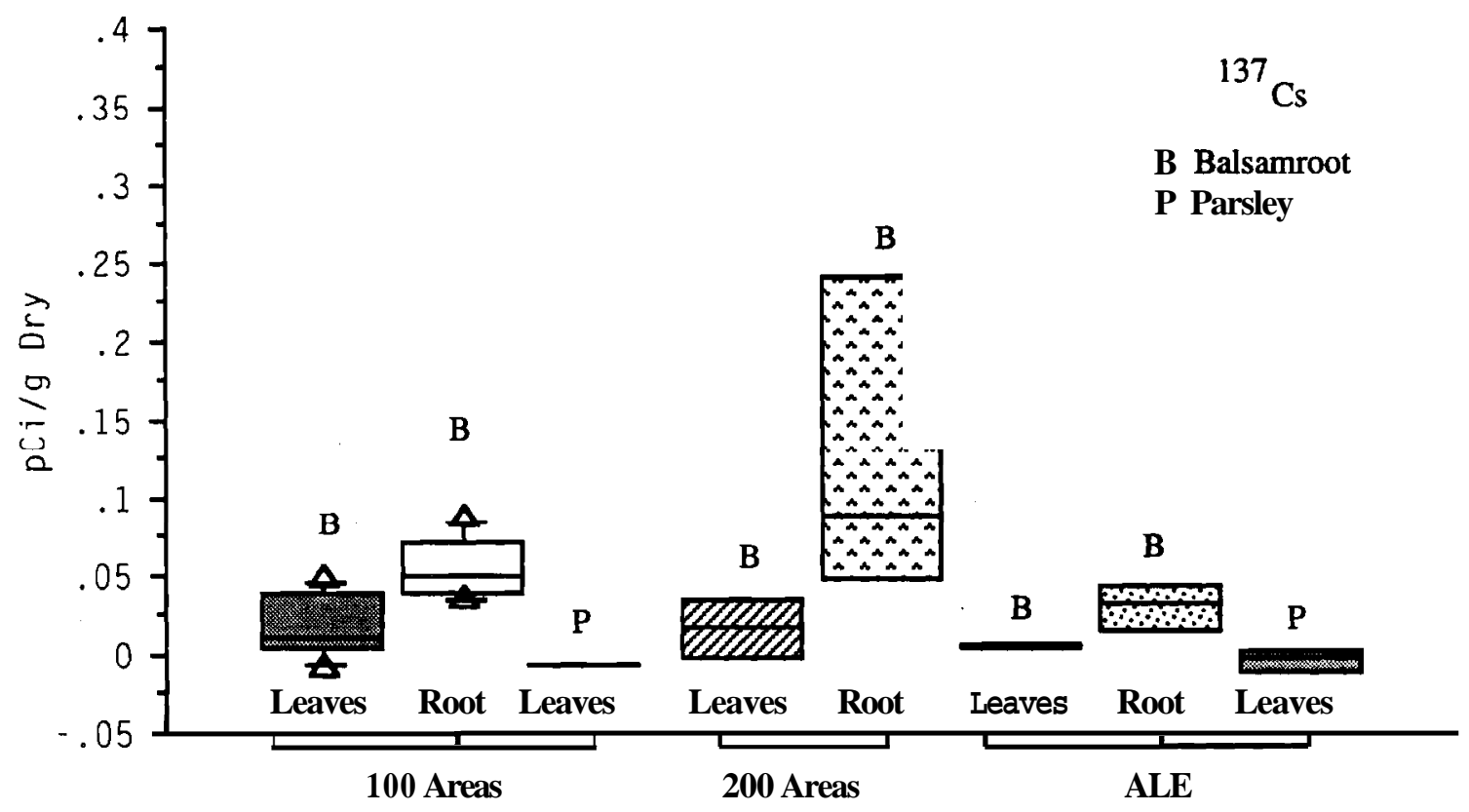

Figure_2 Box Plot of ${ }^{137} \mathrm{Cs}$ in Terrestrial Vegetation

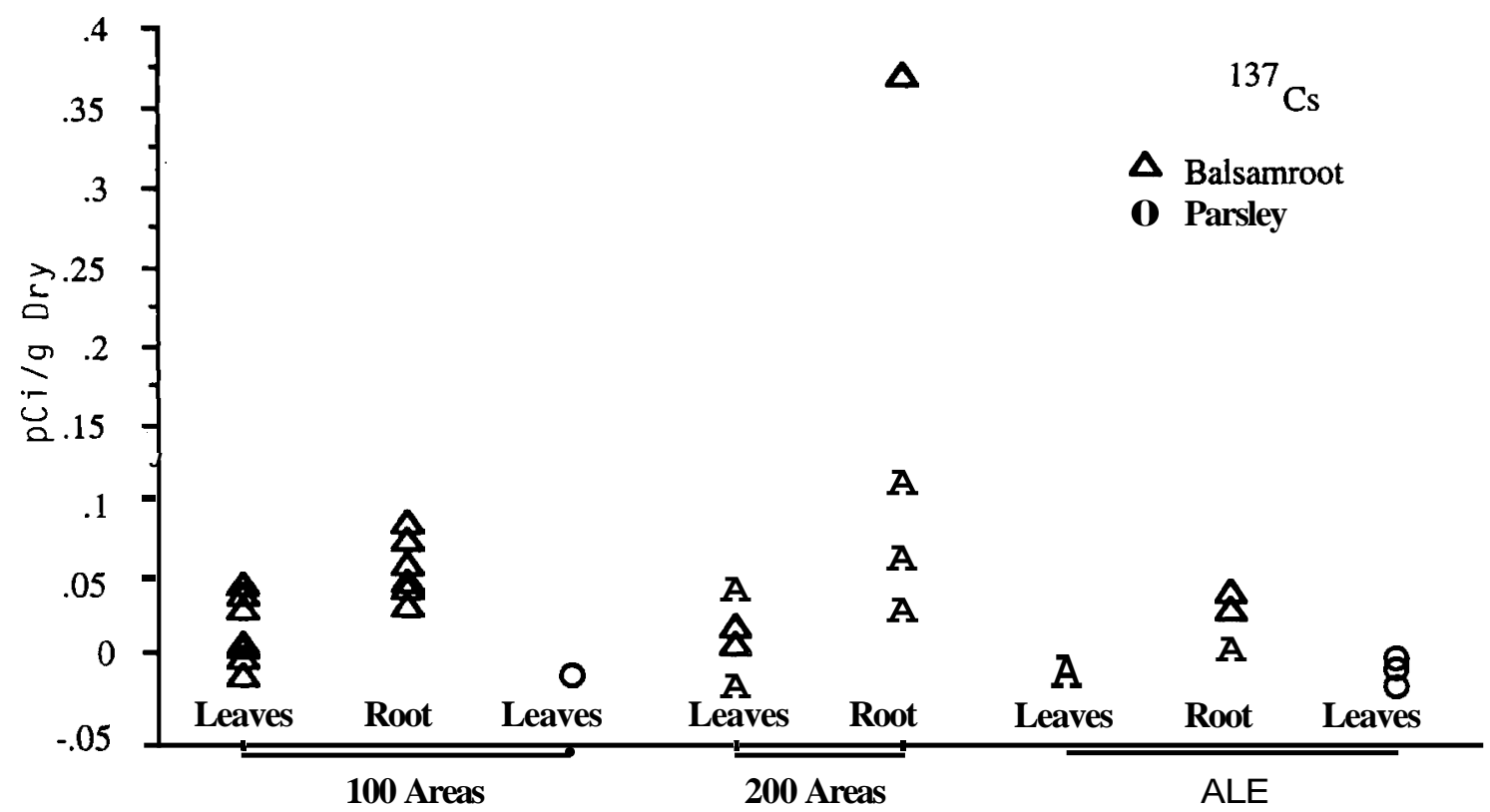

Figure 3. Scattergram of ${ }^{137} \mathrm{Cs}$ in Terrestrial Vegetation 


\section{STRONTIUM-90}

Strontium-90 concentrations in terrestrial vegetation were highest in samples collected from ALE (Figures 4 and 5). As with ${ }^{137} \mathrm{Cs}$, concentrations of ${ }^{90} \mathrm{Sr}$ were higher in root tissue relative to leafy portions. While the concentrations of ${ }^{90} \mathrm{Sr}$ were higher than concentrations of ${ }^{137} \mathrm{Cs}$, the concentrations were within the range of ${ }^{90} \mathrm{Sr}$ associated with other offsite locations that are routinely monitored and reported in annual Site surveillance reports. The maximum and median onsite concentration of ${ }^{90} \mathrm{Sr}$ in terrestrial vegetation sampled onsite from 1985 through 1993 was $8.22( \pm 0.09) \mathrm{pCi} / \mathrm{g}$ (from the 200 Areas), and $0.087 \mathrm{pCi} / \mathrm{g}$ for 88 samples Sitewide, respectively. The maximum and median concentration of ${ }^{90} \mathrm{Sr}$ collected at distant locations was $0.74( \pm 0.02) \mathrm{pCi} / \mathrm{g}$ at Moses Lake, and $0.03 \mathrm{pCi} / \mathrm{g}$ for the 32 samples collected at Washtucna, Moses Lake, Walla Walla, McNary Dam, Sunnyside, and Yakima, respectively.

\section{PERSPECTIVE ON RESULTS}

These data provide an initial indication of concentrations of radionuclides expected in Site terrestrial vegetation of potential interest to Native American people. For ${ }^{90} \mathrm{Sr}$ and ${ }^{137} \mathrm{Cs}$, the concentrations represent contributions from fallout associated with atmospheric weapons testing and possible Hanford Site activities, particularly in samples collected near the industrialized areas. This observation is supported by the similarity in concentrations reported in other natural vegetation collected onsite and offsite in the past. However, the concentrations reported here are very low and where contributions from fallout for ${ }^{90} \mathrm{Sr}$ were comparatively elevated in the ALE samples, elevated ${ }^{137} \mathrm{Cs}$ would also be expected.

No attempt has been made to evaluate the dose that might result from the use of these plants by humans, however, the concentrations are very low and the associated doses associated with plant use are expected to be immeasurable. Information on amounts used, for what purpose the plant is used, and specific parts used are needed to develop dose estimates and are beyond the scope of this initial study. 


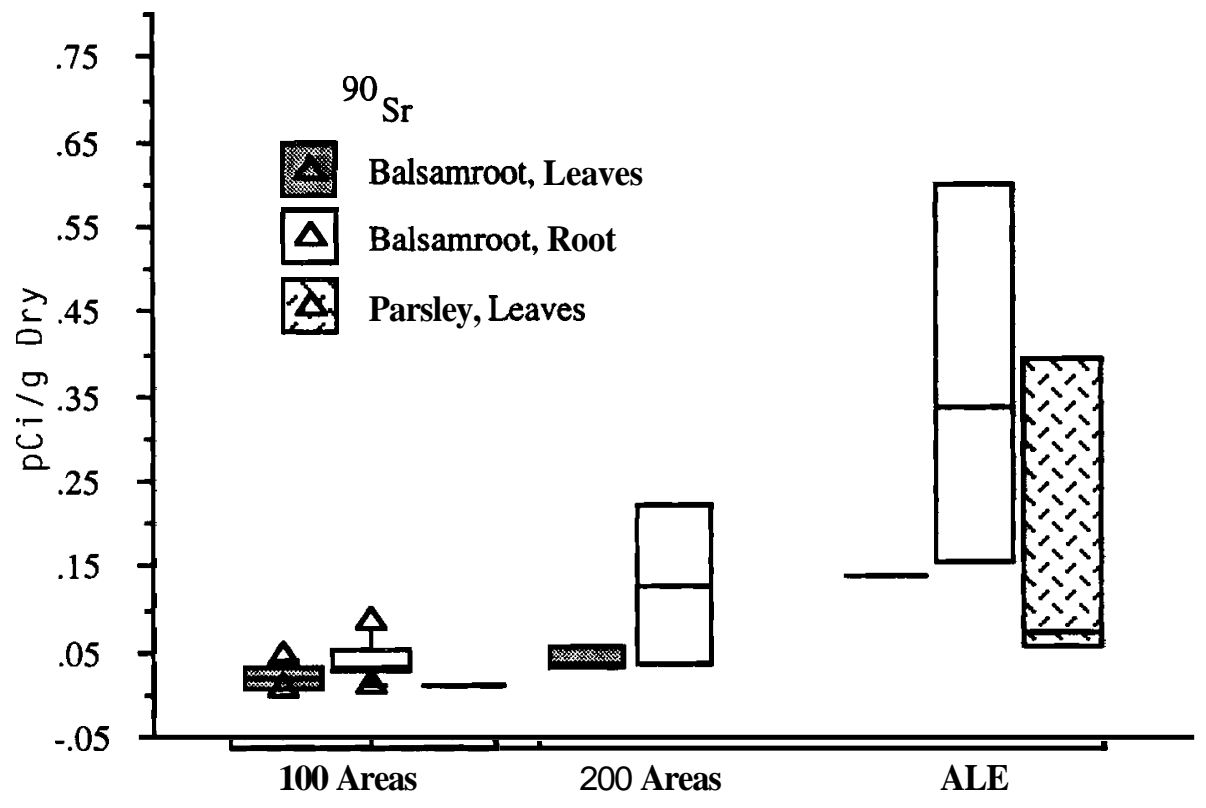

Figure 4. Box Plot of ${ }^{90} \mathrm{Sr}$ in Terrestrial Vegetation

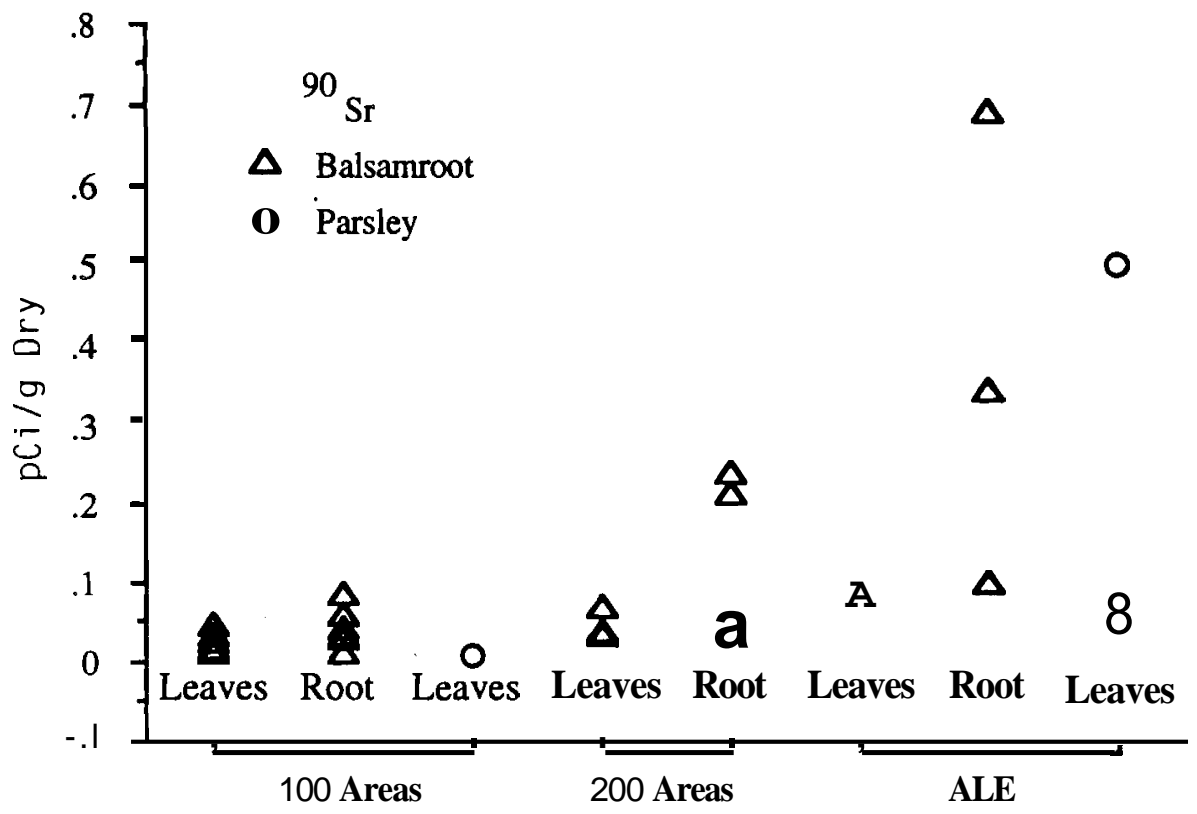

Figure 5. Scattergram of ${ }^{90} \mathrm{Sr}$ in Terrestrial Vegetation 


\section{REFERENCES}

Antonio, E. J., T. M. Poston, and W. H. Rickard. 1993. Radiological Survey of Shoreline Vegetation from the Hanford Reach of the Columbia River, 1990-1992. PNL-8797, Pacific Northwest Laboratory, Richland, Washington.

Daubenmire, R. 1970. Steppe Vegetation of Washington. Tech. Bull. 62. Washington Agricultural Experimental Station, Washington State University, Pullman, Washington.

Downs, J. L., W. H. Rickard, C. A. Brandt, L. L. Cadwell, C. E. Cushing, D. R. Geist, R. M. Mazaika, D. A. Neitzel, L. E. Rogers, M. R. Sackschewsky, and J. J. Nugent. 1993. Habitat Types on the Hanford Site: Wildlife and Plant Species of Concern. PNL-8942, Pacific Northwest Laboratory, Richland, Washington.

Hanford Federal Facility Agreement and Consent Order. 1989 (Tri-Party Agreement) (as amended). 89-10, Rev. 1, Washington State Department of Ecology, U.S. Environmental Protection Agency, and the U.S. Department of Energy.

Rickard, W. H., and B. E. Vaughan. 1988. "Plant Communities: Characteristics and Responses." In Shrub-Steppe Balance and Change in a Semi-Arid Terrestrial Ecosystem, W. H. Rickard, L. E.

Rogers, B. E. Vaughan and S. F. Liebetrau, eds., pp. 109-179. Elsevier Science Publishing Company, Inc., New York.

Sackschewsky, M. R., D. S. Landeen, J. L. Downs, W. H. Rickard, G. I Baird. 1992. Vascular Plants of the Hanford Site. WHC-EP-0554, Westinghouse Hanford Company, Richland, Washington.

Woodruff, R. K., R. W. Hanf, and R. E. Lundgren (eds.). 1993. Hanford Site Environmental Report for Calendar Year 1992. PNL-8682, Pacific Northwest Laboratory, Richland, Washington. 
APPENDIX A

SUMMARY STATISTICS 
Table A.1. Summary Statistics (pCi/g dry) for Terrestrial Vegetation Collected on the Hanford Site in 1994

\begin{tabular}{|c|c|c|c|c|c|c|c|c|c|c|c|c|c|c|}
\hline adionuclide & MDC & Location & Species & Portion & Median & Mean & $\begin{array}{l}\text { Standard } \\
\text { Deviation } \\
\end{array}$ & $\begin{array}{c}\text { Standard } \\
\text { Error }\end{array}$ & Count & Minimum & Maximum & Variance & $\begin{array}{l}\text { Coefficient } \\
\text { of Variation } \\
\end{array}$ & $\begin{array}{c}\text { Geometric } \\
\text { Mean }\end{array}$ \\
\hline $\mathrm{Be} 7$ & 0.23 & 100 Areas & Balsamroot & Leaves & 2.2000 & 2.2771 & 0.8305 & 0.3139 & 7 & 1.3700 & 3.6700 & 0.6897 & 0.365 & 2.153 \\
\hline $\mathrm{Be} 7$ & 0.23 & 100 Areas & alsamroot & Root & 0.0284 & 0.0891 & 0.1243 & 0.0470 & 7 & -0.0434 & 0.2780 & 0.0155 & 1.395 & NV \\
\hline Be7 & 0.23 & 100 Areas & Cymopterus & Leaves & 4.0400 & 4.0400 & $\mathrm{NV}$ & & 1 & 4.0400 & 4.0400 & $\mathrm{NV}$ & $\mathrm{NV}$ & 40 \\
\hline $\mathrm{Be}-7$ & 0.23 & 200 Areas & Balsamroot & Leaves & 1.2950 & 1.2850 & 0.3361 & 0.1681 & 4 & 0.8900 & 1.6600 & 0.1130 & 0.262 & 1.25 \\
\hline $\mathrm{Be} 7$ & 0.23 & 200 Areas & Balsamroot & Root & 0.0591 & 0.0186 & 0.0886 & 0.0443 & 4 & -0.1140 & 0.0702 & 0.0079 & 4.769 & $\mathrm{NV}$ \\
\hline Be-7 & 0.23 & ALE & Balsamroot & Leaves & 1.5200 & 1.8500 & 0.6243 & 0.3604 & 3 & 1.4600 & 2.5700 & 0.3897 & 0.337 & 1.78 \\
\hline Be7 & 0.23 & ALE & Balsamroot & Root & 0.0347 & 0.0528 & 0.0680 & 0.0393 & 3 & -0.0043 & 0.1280 & 0.0046 & 1.288 & $\mathrm{NV}$ \\
\hline $\mathrm{Be} 7$ & 0.23 & ALE & Lomatium & Leaves & 3.2600 & 3.1300 & 0.3440 & 0.1986 & 3 & 2.7400 & 3.3900 & 0.1183 & 0.110 & 3.1 \\
\hline Co-60 & 0.03 & 100 Areas & Balsamroot & Leaves & 0.0010 & -0.0003 & 0.0278 & 0.0105 & 7 & -0.0515 & 0.0438 & 0.0008 & -100.366 & $\mathrm{NV}$ \\
\hline Co-60 & 0.03 & 100 Areas & Balsamroot & Root & 0.0000 & 0.0086 & 0.0194 & 0.0074 & 7 & -0.0093 & 0.0441 & 0.0004 & 2.257 & $\mathrm{NV}$ \\
\hline Co-60 & 0.03 & 100 Areas & Cymopterus & Leaves & 0.0120 & 0.0120 & NV & $\mathrm{NV}$ & 1 & 0.0120 & 0.0120 & $\mathrm{NV}$ & $\mathrm{NV}$ & 0.01 \\
\hline Co-60 & 0.03 & 200 Areas & Balsamroot & Leaves & . -0.0138 & -0.0134 & 0.0063 & 0.0032 & 4 & -0.0206 & -0.0053 & 0.0000 & -0.472 & $\mathrm{NV}$ \\
\hline Co-60 & 0.03 & 200 Areas & Balsamroot & Root & 0.0012 & 0.0017 & 0.0039 & 0.0020 & 4 & -0.0024 & 0.0069 & 0.0000 & 2.251 & $\mathrm{NV}$ \\
\hline Co-60 & 0.03 & ALE & Balsamroot & Leaves & -0.0134 & -0.0164 & 0.0127 & 0.0073 & 3 & -0.0303 & -0.0056 & 0.0002 & -0.770 & NV \\
\hline Co-60 & 0.03 & ALE & Balsamroot & Root & -0.0037 & -0.0039 & 0.0085 & 0.0049 & 3 & -0.0124 & 0.0045 & .0001 & -2.175 & NV \\
\hline Co-60 & 0.03 & & & & -0.0138 & -0.0097 & 0.0079 & 0.0045 & 3 & -0.0147 & -0.0007 & 0.0001 & -0.808 & NV \\
\hline Cs-137 & 0.03 & 100 Areas & Balsamroot & Leaves & 0.0114 & 0.0193 & 0.0209 & 0.0079 & 7 & -0.0078 & 0.0468 & 0.0004 & 1.082 & NV \\
\hline Cs-137 & 0.03 & 100 Areas & Balsamroot & Root & 0.0503 & 0.0555 & 0.0200 & 0.0075 & 7 & 0.0340 & 0.0870 & 0.0004 & 0.360 & 0.05 \\
\hline Cs- 137 & 0.03 & 100 Areas & Cymopterus & Leaves & -0.0037 & -0.0037 & $\mathrm{NV}$ & NV & 1 & -0.0037 & -0.0037 & $\mathrm{NV}$ & $\mathrm{NV}$ & NV \\
\hline Cs-137 & 0.03 & 00 Arcas & alsamroot & Leaves & 0.0166 & 0.0161 & 0.0253 & 0.0127 & 4 & 148 & 0.0459 & 006 & 1.576 & NV \\
\hline & 0.03 & 200 Areas & & & 0.0900 & 0.1451 & & 0.0765 & 4 & & 0.3690 & 0.0234 & 1.054 & $0.0 s$ \\
\hline Cs-137 & 0.03 & ALE & Balsamroot & Leaves & 0.0047 & 0.0053 & 0.0027 & 0.0016 & 3 & 0.0029 & 0.0082 & 0.0000 & 0.508 & 0.00 \\
\hline Cs-137 & 0.03 & ALE & Balsamroot & Root & 0.0321 & 0.0299 & 0.0195 & 0.0112 & 3 & 0.0094 & 0.0481 & 0.0004 & 0.652 & 0.02 \\
\hline Cs-137 & 0.03 & ALE & Lomatium & Leaves & -0.0020 & -0.0041 & 0.0087 & 0.0050 & 3 & -0.0136 & 0.0034 & 0.0001 & -2.147 & $\mathrm{NV}$ \\
\hline & & & & eaves & 34.8000 & & & & 7 & & 39.9000 & 14.8867 & 0.112 & 34.105 \\
\hline K 40 & & 100 Areas & & & 7.0500 & 7.4557 & & 0.6115 & 7 & & 10.9000 & 2.6177 & 0.217 & 7.32 \\
\hline $\mathrm{K} 40$ & 0.53 & 100 Areas & ymoptems & Leaves & 32.8000 & 32.8000 & & & 1 & & 32.8000 & $\mathrm{NV}$ & $\mathrm{NV}$ & 32.80 \\
\hline $\mathrm{K}-40$ & 0.53 & 200 Areas & Balsamroot & Leaves & 42.1000 & 43.5750 & 3.3009 & 1.6504 & 4 & 41.6000 & 48.5000 & 10.8958 & 0.076 & 43.485 \\
\hline K40 & 0.53 & 200 Areas & Balsamroot & Root & 8.1850 & 8.1750 & 0.0889 & 0.0444 & 4 & 8.0600 & 8.2700 & 0.0079 & 0.011 & 8.175 \\
\hline K-40 & 0 & ALE & & Leaves & 30.9000 & 31.1667 & 4.5059 & 2.6015 & 3 & 26.8000 & 35.8000 & 20.3033 & 0.145 & 30.950 \\
\hline $\mathrm{K}-40$ & & ALE & Balsamroot & & 5.8800 & 5.6833 & 0.5046 & 0.2913 & 3 & 5.1100 & 6.0600 & 0.2546 & 0.089 & 5.668 \\
\hline & & ALE & & Leaves & 38.6000 & 41.2000 & & 2.6502 & 3 & 38.5000 & 46.5000 & 21.0700 & 0.111 & 41.036 \\
\hline Sr-90 & 0.005 & 100 Areas & Balsamroot & Leaves & 0.0217 & 0.0218 & 0.0139 & 0.0053 & 7 & 0.0069 & 0.0444 & 0.0002 & 0.638 & 0.018 \\
\hline Sr-90 & 0.005 & 100 Areas & Balsamroot & Root & 0.0317 & 0.0397 & 0.0244 & 0.0092 & 7 & 0.0097 & 0.0847 & 0.0006 & 0.615 & 0.033 \\
\hline Sr-90 & 0.005 & 100 Areas & Cymopterus & Leaves & 0.0074 & 0.0074 & $\mathrm{NV}$ & $\mathrm{NV}$ & 1 & 0.0074 & 0.0074 & $\mathrm{NV}$ & $\mathrm{NV}$ & 0.007 \\
\hline Sr-90 & 0.005 & 200 Areas & Balsamroot & Leaves & 0.0342 & 0.0426 & 0.0187 & 0.0108 & 3 & 0.0296 & 0.0641 & 0.0004 & 0.439 & 0.04 \\
\hline Sr-90 & 0.005 & 200 Areas & Balsamroot & Root & 0.1261 & 0.1292 & 0.1068 & 0.0534 & 4 & 0.0296 & 0.2350 & 0.0114 & 0.827 & 0.090 \\
\hline Sr-90 & 0.005 & ALE & Balsamroot & Leaves & 0.0810 & 0.0810 & $\mathrm{NV}$ & $\mathrm{NV}$ & 1 & 0.0810 & 0.0810 & $\mathrm{NV}$ & $\mathrm{NV}$ & 0.081 \\
\hline Sr-90 & 0.005 & ALE & & & 0.3380 & 0.3740 & 0.2976 & 0.1718 & 3 & 0.0961 & 0.6880 & 0.0886 & 0.796 & 0.282 \\
\hline Sf-90 & 0.005 & ALE & Lomatium & Leaves & 0.0737 & 0.2082 & 0.2512 & 0,1450 & 3 & 0.0530 & 0.4980 & 0.0631 & 1.206 & 0.125 \\
\hline
\end{tabular}

$\mathrm{NV}=$ No value

Values good to two decimal places only for gamma spectroscopy results, three decimal places for Strontium- 90 values. 
APPENDIX B

ANALYTICAL RESULTS 
Table B.1. Analytical Results (pCi/g dry) of Vegetation Samples

\begin{tabular}{|c|c|c|c|c|c|c|c|c|c|c|}
\hline $\begin{array}{l}\text { Sample } \\
\text { Number }\end{array}$ & Plant ID & Location & Plant Species & Sample & Constituent & $\begin{array}{c}\text { Date } \\
\text { (Y/M/D) }\end{array}$ & Result & $\begin{array}{l}\text { Counting } \\
\text { Error }\end{array}$ & $\begin{array}{c}\text { Overall } \\
\text { Error }\end{array}$ & $\begin{array}{l}\text { Analysis } \\
\text { Size (g) }\end{array}$ \\
\hline B0BW61 & B-1 & ALE & Balsamroot & Leaves & $\mathrm{Be}-7$ & 940428 & $2.57 \mathrm{E}+00$ & $3.84 \mathrm{E}-01$ & $4.62 \mathrm{E}-01$ & 161 \\
\hline B0BW61 & B-1 & ALE & Balsamroot & Leaves & $\mathrm{Co}-60$ & 940428 & $-1.34 \mathrm{E}-02$ & $3.72 \mathrm{E}-02$ & $3.72 \mathrm{E}-02$ & 161 \\
\hline B0BW61 & B-1 & ALE & Balsamroot & Leaves & Cs- 137 & 940428 & $8.20 \mathrm{E}-03$ & $2.83 \mathrm{E}-02$ & $2.83 \mathrm{E}-02$ & 161 \\
\hline B0BW61 & B-1 & ALE & Balsamroot & Leaves & $\mathbf{K}-40$ & 940428 & $2.68 \mathrm{E}+01$ & $1.27 \mathrm{E}+00$ & $2.97 \mathrm{E}+00$ & 161 \\
\hline B0BW61 & B-1 & ALE & Balsamroot & Leaves & $\mathrm{Sr}-90$ & 940428 & $8.10 \mathrm{E}-02$ & $1.20 \mathrm{E}-02$ & $1.88 \mathrm{E}-02$ & 104 \\
\hline B0BW62 & B-1 & ALE & Balsamroot & Root & $\mathrm{Be}-7$ & 940428 & $1.28 \mathrm{E}-01$ & $1.03 \mathrm{E}-01$ & $1.04 \mathrm{E}-01$ & 229 \\
\hline B0BW62 & B-1 & ALE & Balsamroot & Root & $\mathrm{Co}-60$ & 940428 & $-1.24 \mathrm{E}-02$ & $1.24 \mathrm{E}-02$ & $1.25 \mathrm{E}-02$ & 229 \\
\hline B0BW62 & B-1 & ALE & Balsamroot & Root & Cs- 137 & 940428 & $4.81 \mathrm{E}-02$ & $1.37 \mathrm{E}-02$ & $1.45 \mathrm{E}-02$ & 229 \\
\hline B0BW62 & B-1 & ALE & Balsamroot & Root & $\mathbf{K}-40$ & 940428 & $6.06 \mathrm{E}+00$ & $3.83 \mathrm{E}-01$ & $7.17 \mathrm{E}-01$ & 229 \\
\hline B0BW62 & B-1 & ALE & Balsamroot & Root & Sr -90 & 940428 & $6.88 \mathrm{E}-01$ & $2.37 \mathrm{E}-02$ & $1.27 \mathrm{E}-01$ & 100 \\
\hline B0BW63 & P-1 & ALE & Lomation & Leaves & $\mathrm{Be}-7$ & 940428 & $3.26 \mathrm{E}+00$ & $5.48 \mathrm{E}-01$ & $6.37 \mathrm{E}-01$ & 133 \\
\hline В0ВW63 & P-1 & ALE & Lomation & Leaves & $\mathrm{Co}-60$ & 940428 & $-1.38 \mathrm{E}-02$ & $4.32 \mathrm{E}-02$ & $4.32 \mathrm{E}-02$ & 133 \\
\hline B0BW63 & P-1 & ALE & Lomation & Leaves & Cs- 137 & 940428 & $-1.36 \mathrm{E}-02$ & $3.52 \mathrm{E}-02$ & $3.53 \mathrm{E}-02$ & 133 \\
\hline B0BW63 & P-1 & ALE & Lomation & Leaves & $\mathbf{K}-40$ & 940428 & $3.86 \mathrm{E}+01$ & $1.65 \mathrm{E}+00$ & $4.20 \mathrm{E}+00$ & 133 \\
\hline B0BW63 & P-1 & ALE & Lomation & Leaves & Sr -90 & 940428 & $4.98 \mathrm{E}-01$ & $3.98 \mathrm{E}-02$ & $9.82 \mathrm{E}-02$ & 102 \\
\hline B0BW64 & P-2 & ALE & Lomation & Leaves & $\mathrm{Be}-7$ & 940428 & $3.39 \mathrm{E}+00$ & $3.90 \mathrm{E}-01$ & $5.17 \mathrm{E}-01$ & 138 \\
\hline B0BW64 & $\mathrm{P}-2$ & ALE & Lomation & Leaves & Co- 60 & 940428 & $-1.47 \mathrm{E}-02$ & $2.26 \mathrm{E}-02$ & $2.27 \mathrm{E}-02$ & 138 \\
\hline B0BW64 & P-2 & ALE & Lomation & Leaves & Cs- 137 & 940428 & $-1.99 \mathrm{E}-03$ & $1.73 \mathrm{E}-02$ & $1.73 \mathrm{E}-02$ & 138 \\
\hline B0BW64 & $\mathrm{P}-2$ & ALE & Lomation & Leaves & $\mathrm{K}-40$ & 940428 & $4.65 \mathrm{E}+01$ & $1.31 \mathrm{E}+00$ & $4.83 \mathrm{E}+00$ & 138 \\
\hline B0BW64 & $P-2$ & ALE & Lomation & Leaves & Sr -90 & 940428 & $7.37 \mathrm{E}-02$ & $1.14 \mathrm{E}-02$ & $1.79 \mathrm{E}-02$ & 100 \\
\hline B0BW65 & P-3 & ALE & Lomation & Leaves & $\mathrm{Be}-7$ & 940428 & $2.74 \mathrm{E}+00$ & $2.55 \mathrm{E}-01$ & $3.74 \mathrm{E}-01$ & 173 \\
\hline B0BW65 & P-3 & ALE & Lomation & Leaves & Co-60 & 940428 & $-6.72 \mathrm{E}-04$ & $2.24 \mathrm{E}-02$ & $2.24 \mathrm{E}-02$ & 173 \\
\hline B0BW65 & P-3 & ALE & Lomation & Leaves & Cs- 137 & 940428 & $3.43 \mathrm{E}-03$ & $1.60 \mathrm{E}-02$ & $1.60 \mathrm{E}-02$ & 173 \\
\hline B0BW65 & P-3 & ALE & Lomation & Leaves & K-40 & 940428 & $3.85 \mathrm{E}+01$ & $1.05 \mathrm{E}+00$ & $3.99 \mathrm{E}+00$ & 173 \\
\hline B0BW65 & P-3 & ALE & Lomation & Leaves & $\mathrm{Sr}-90$ & 940428 & $5.30 \mathrm{E}-02$ & 8.37E-03 & $1.28 \mathrm{E}-02$ & 100 \\
\hline B0BW66 & B-2 & ALE & Balsamroot & Root & $\mathrm{Be}-7$ & 940428 & $-4.32 \mathrm{E}-03$ & $7.26 \mathrm{E}-02$ & $7.26 \mathrm{E}-02$ & 321 \\
\hline B0BW66 & B-2 & ALE & Balsamroot & Root & $\mathrm{Co}-60$ & 940428 & $-3.74 \mathrm{E}-03$ & $8.38 \mathrm{E}-03$ & $8.39 \mathrm{E}-03$ & 321 \\
\hline B0BW66 & B-2 & ALE & Balsamroot & Root & Cs-137 & 940428 & $3.21 \mathrm{E}-02$ & $1.10 \mathrm{E}-02$ & $1.15 \mathrm{E}-02$ & 321 \\
\hline B0BW66 & B-2 & ALE & Balsamroot & Root & $\mathrm{K}-40$ & 940428 & $5.88 \mathrm{E}+00$ & $3.57 \mathrm{E}-01$ & $6.87 \mathrm{E}-01$ & 321 \\
\hline B0BW66 & B-2 & ALE & Balsamroot & Root & Sr -90 & 940428 & $3.38 \mathrm{E}-01$ & $2.17 \mathrm{E}-02$ & $8.61 \mathrm{E}-02$ & 106 \\
\hline B0BW67 & B-2 & ALE & Balsamroot & Leaves & $\mathrm{Be}-7$ & 940428 & $1.46 \mathrm{E}+00$ & $2.27 \mathrm{E}-01$ & $2.70 \mathrm{E}-01$ & 159 \\
\hline B0BW67 & B-2 & ALE & Balsamroot & Leaves & $\mathrm{Co}-60$ & 940428 & $-3.03 \mathrm{E}-02$ & $2.46 \mathrm{E}-02$ & $2.48 \mathrm{E}-02$ & 159 \\
\hline B0BW67 & B-2 & ALE & Balsamroot & Leaves & Cs-137 & 940428 & $2.92 \mathrm{E}-03$ & $1.69 \mathrm{E}-02$ & $1.70 \mathrm{E}-02$ & 159 \\
\hline B0BW67 & B-2 & ALE & Balsamroot & Leaves & K-40 & 940428 & $3.09 \mathrm{E}+01$ & $1.00 \mathrm{E}+00$ & $3.25 \mathrm{E}+00$ & 159 \\
\hline B0BW67 & B-2 & ALE & Balsamroot & Leaves & Sr -90 & 940428 & & & & \\
\hline B0BW68 & B-3 & ALE & Balsamroot & Root & $\mathrm{Be}-7$ & 940428 & $3.47 \mathrm{E}-02$ & 7.35E-02 & $7.36 \mathrm{E}-02$ & 296 \\
\hline B0BW68 & B-3 & ALE & Balsamroot & Root & Co-60 & 940428 & $4.49 \mathrm{E}-03$ & 7.62E-03 & $7.64 \mathrm{E}-03$ & 296 \\
\hline B0BW68 & B-3 & ALE & Balsamroot & Root & Cs- 137 & 940428 & $9.37 \mathrm{E}-03$ & $7.38 \mathrm{E}-03$ & 7.44E-03 & 296 \\
\hline B0BW68 & B-3 & ALE & Balsamroot & Root & $\mathrm{K}-40$ & 940428 & $5.11 \mathrm{E}+00$ & $3.20 \mathrm{E}-01$ & $6.03 \mathrm{E}-01$ & 296 \\
\hline B0BW68 & B-3 & ALE & Balsamroot & Root & $\mathrm{Sr}-90$ & 940428 & $9.61 \mathrm{E}-02$ & $9.34 \mathrm{E}-03$ & $2.44 \mathrm{E}-02$ & 107 \\
\hline B0BW69 & B-3 & ALE & Balsamroot & Leaves & $\mathrm{Be}-7$ & 940428 & $1.52 \mathrm{E}+00$ & $2.92 \mathrm{E}-01$ & $3.29 \mathrm{E}-01$ & 199 \\
\hline B0BW69 & B-3 & ALE & Balsamroot & Leaves & Co-60 & 940428 & $-5.55 \mathrm{E}-03$ & $1.90 \mathrm{E}-02$ & $1.90 \mathrm{E}-02$ & 199 \\
\hline B0BW69 & B-3 & ALE & Balsamroot & Leaves & Cs- 137 & 940428 & $4.73 \mathrm{E}-03$ & $1.29 \mathrm{E}-02$ & $1.29 \mathrm{E}-02$ & 199 \\
\hline
\end{tabular}


Table B.1. (con't)

\begin{tabular}{|c|c|c|c|c|c|c|c|c|c|c|}
\hline $\begin{array}{l}\text { Sample } \\
\text { Number }\end{array}$ & Plant ID & Location & Plant Species & Sample & Constituent & $\begin{array}{c}\text { Date } \\
\text { (Y/M/D) }\end{array}$ & Result & $\begin{array}{c}\text { Counting } \\
\text { Error }\end{array}$ & $\begin{array}{c}\text { Overall } \\
\text { Error }\end{array}$ & $\begin{array}{l}\text { Analysis } \\
\text { Size (g) }\end{array}$ \\
\hline B0BW69 & B-3 & ALE & & Leaves & $\mathrm{K}-40$ & 940428 & $3.58 \mathrm{E}+01$ & $9.84 \mathrm{E}-01$ & $3.71 \mathrm{E}+00$ & 199 \\
\hline B0BW69 & B-3 & ALE & & Leaves & Sr -90 & 940428 & & & & \\
\hline B0BW70 & B-4 & 200 Areas & Balsamroot & Leaves & $\mathrm{Be}-7$ & 940428 & $8.90 \mathrm{E}-01$ & $2.36 \mathrm{E}-01$ & $2.52 \mathrm{E}-01$ & 152 \\
\hline BOBW70 & B-4 & 200 Areas & Balsamroot & Leaves & $\mathrm{Co}-60$ & 40428 & $-2.06 \mathrm{E}-02$ & $2.78 \mathrm{E}-02$ & $2.79 \mathrm{E}-02$ & 152 \\
\hline BOBW70 & B-4 & 200 Areas & Balsamroot & Leaves & Cs- 137 & 940428 & $1.04 \mathrm{E}-02$ & $1.95 \mathrm{E}-02$ & $1.96 \mathrm{E}-02$ & 152 \\
\hline B0BW70 & B-4 & 200 Areas & Balsamroot & Leaves & $\mathrm{K}-40$ & 940428 & $4.85 \mathrm{E}+01$ & $1.26 \mathrm{E}+00$ & $5.01 \mathrm{E}+00$ & 152 \\
\hline BOBW70 & B-4 & 200 Areas & Balsamroot & Leaves & Sr -90 & 940428 & $2.96 \mathrm{E}-02$ & $6.13 \mathrm{E}-03$ & $8.21 \mathrm{E}-03$ & 1.15 \\
\hline BOBW71 & B-4 & 200 Areas & Balsamroot & Root & $\mathrm{Be}-7$ & & $7.02 \mathrm{E}-02$ & $1.01 \mathrm{E}-01$ & $1 \mathrm{E}-01$ & 218 \\
\hline B0BW71 & B-4 & 200 Areas & Balsamroot & Root & $\mathrm{Co}-60$ & 940428 & $2.16 \mathrm{E}-03$ & $1.06 \mathrm{E}-02$ & $1.06 \mathrm{E}-02$ & 218 \\
\hline B0BW71 & B-4 & 200 Areas & Balsamroot & Root & Cs- 137 & 940428 & $1.14 \mathrm{E}-01$ & $2.04 \mathrm{E}-02$ & $2.34 \mathrm{E}-02$ & 218 \\
\hline B0BW71 & B-4 & 200 Areas & Balsamroot & Root & & 940428 & $8.27 \mathrm{E}+00$ & $5.07 \mathrm{E}-01$ & $9.70 \mathrm{E}-01$ & 218 \\
\hline B0BW71 & B-4 & 200 Areas & Balsamroot & Root & Sr-90 & 940428 & $2.07 \mathrm{E}-01$ & $1.17 \mathrm{E}-02$ & $4.05 \mathrm{E}-02$ & 106 \\
\hline B0BW72 & B-5 & 200 Areas & Balsamroot & Leaves & $\mathrm{Be}-7$ & 940428 & $1.66 \mathrm{E}+00$ & 4.42E-01 & E-01 & 176 \\
\hline B0BW72 & B-5 & 200 Areas & Balsamroot & Leaves & Co-60 & 940428 & $-1.28 \mathrm{E}-02$ & $3.02 \mathrm{E}-02$ & $3.02 \mathrm{E}-02$ & 176 \\
\hline B0BW72 & B-5 & 200 Areas & Balsamroot & & & 940428 & $2.27 \mathrm{E}-02$ & $2.49 \mathrm{E}-02$ & E-02 & 176 \\
\hline B0BW72 & B-5 & 200 Areas & Balsamroot & Leaves & $\mathrm{K}-40$ & 940428 & $4.24 \mathrm{E}+01$ & $1.42 \mathrm{E}+00$ & $4.47 \mathrm{E}+00$ & 176 \\
\hline BOBW72 & B-5 & & Balsamroot & & & 940428 & $3.42 \mathrm{E}-02$ & $5.90 \mathrm{E}-03$ & 8.77E-03 & 101 \\
\hline BOBW73 & B-5 & 200 Areas & Balsamroot & Root & $\mathrm{Be}-7$ & 940428 & $6.28 \mathrm{E}-02$ & $9.37 \mathrm{E}-02$ & $9.39 \mathrm{E}-02$ & 294 \\
\hline B0BW73 & B-5 & 200 Areas & Balsamroot & & Co-60 & 940428 & $6.91 \mathrm{E}-03$ & $1.01 \mathrm{E}-02$ & E-02 & 294 \\
\hline B0BW73 & B-5 & 200 Areas & Balsamroot & Root & Cs- 137 & 940428 & $6.59 \mathrm{E}-02$ & $1.22 \mathrm{E}-02$ & $1.39 \mathrm{E}-02$ & 294 \\
\hline B0BW73 & B-5 & 200 Areas & Balsamroot & Root & & 0428 & $8.16 \mathrm{E}+00$ & E-01 & & 294 \\
\hline B0BW73 & B-5 & 200 Areas & Balsamroot & Root & Sr-90 & 940428 & $4.51 \mathrm{E}-02$ & $9.71 \mathrm{E}-03$ & $1.27 \mathrm{E}-02$ & 104 \\
\hline B0BW74 & B-6 & 200 Areas & Balsamroot & Leaves & & 0428 & $1.15 \mathrm{E}+00$ & 2.5 & & 204 \\
\hline B0BW74 & B-6 & 200 Areas & Balsamroot & Leaves & Co- 60 & 940428 & $-1.47 \mathrm{E}-02$ & $1.86 \mathrm{E}-02$ & E-02 & 204 \\
\hline B0BW74 & B-6 & 200 Areas & Balsamroot & Leaves & Cs-137 & 40428 & $-1.48 \mathrm{E}-02$ & 1E-02 & & 204 \\
\hline B0BW74 & B-6 & 200 Areas & Balsamroot & Leaves & $\mathrm{K}-40$ & 940428 & $4.18 \mathrm{E}+01$ & $1.04 \mathrm{E}+00$ & $4.31 \mathrm{E}+00$ & 204 \\
\hline B0BW74 & B-6 & 200 Areas & Balsamroot & Leaves & Sr-90 & 940428 & & & & \\
\hline B0BW75 & B-6 & $200 \mathrm{~A}$ & & & $\mathrm{Be}-7$ & 940428 & $-1.14 \mathrm{E}-01$ & $1.03 \mathrm{E}-01$ & $4 \mathrm{E}-01$ & 252 \\
\hline B0BW75 & B-6 & 200 Areas & Balsamroot & Root & Co-60 & 940428 & $-2.38 \mathrm{E}-03$ & 9.68 & E-03 & 252 \\
\hline B0BW75 & B-6 & 200 Areas & Balsamroot & Root & Cs- & 0428 & $3.16 \mathrm{E}-02$ & $1.53 \mathrm{E}-02$ & $1.56 \mathrm{E}-02$ & 252 \\
\hline B0BW75 & B-6 & 200 Areas & Balsamroot & Root & $\mathrm{K}-40$ & 940428 & $8.21 \mathrm{E}+00$ & 4.45E-01 & & 252 \\
\hline В0ВW75 & B-6 & 200 Areas & Balsamroot & Root & Sr-90 & 940428 & $2.96 \mathrm{E}-02$ & 1.1 & -02 & 112 \\
\hline B0BW76 & B-7 & 200 Areas & Balsamroot & Root & $\mathrm{Be}-7$ & 940428 & $5.53 \mathrm{E}-02$ & $1.16 \mathrm{E}-01$ & $1.16 \mathrm{E}-01$ & 182 \\
\hline B0BW76 & B-7 & 200 Areas & Balsamroot & Root & Co-60 & 940428 & $2.70 \mathrm{E}-04$ & $1.21 \mathrm{E}-02$ & $1 \mathrm{E}-02$ & 182 \\
\hline B0BW76 & B-7 & 200 Areas & Balsamroot & Root & Cs- 137 & 940428 & $3.69 \mathrm{E}-01$ & $3.02 \mathrm{E}-02$ & $4.77 \mathrm{E}-02$ & 182 \\
\hline B0BW76 & B-7 & 200 Areas & Balsamroot & Root & $\mathrm{K}-40$ & 940428 & $8.06 \mathrm{E}+00$ & $5.19 \mathrm{E}-01$ & $9.58 \mathrm{E}-01$ & 182 \\
\hline B0BW76 & B-7 & 200 Areas & Balsamroot & Root & Sr -90 & 940428 & $2.35 \mathrm{E}-01$ & $2.29 \mathrm{E}-02$ & $4.87 \mathrm{E}-02$ & 102 \\
\hline B0BW77 & B-7 & 200 Areas & Balsamroot & Leaves & $\mathrm{Be}-7$ & 940428 & $1.44 \mathrm{E}+00$ & $3.66 \mathrm{E}-01$ & $3.93 \mathrm{E}-01$ & 121 \\
\hline B0BW77 & B-7 & 200 Areas & Balsamroot & Leaves & Co- 60 & 940428 & $-5.31 \mathrm{E}-03$ & $2.41 \mathrm{E}-02$ & $2.41 \mathrm{E}-02$ & 121 \\
\hline B0BW77 & B-7 & 200 Areas & Balsamroot & Leaves & Cs- 137 & 940428 & $4.59 \mathrm{E}-02$ & $2.26 \mathrm{E}-02$ & $2.30 \mathrm{E}-02$ & 121 \\
\hline BOBW77 & B-7 & 200 Areas & Balsamroot & Leaves & $K-40$ & 940428 & $4.16 \mathrm{E}+01$ & $1.41 \mathrm{E}+00$ & $4.39 \mathrm{E}+00$ & 121 \\
\hline B0BW77 & B-7 & 200 Areas & Balsamroot & Leaves & $\mathrm{Sr}_{\mathrm{r}-90}$ & 940428 & $6.41 \mathrm{E}-02$ & $8.38 \mathrm{E}-03$ & $1.78 \mathrm{E}-02$ & 100 \\
\hline B0BWP3 & B-8 & K-N, 100 Area & Balsamroot & Leaves & $\mathrm{Be}-7$ & 940505 & $2.85 \mathrm{E}+00$ & $5.39 \mathrm{E}-01$ & $6.10 \mathrm{E}-01$ & 82 \\
\hline
\end{tabular}


Table B.1. (con't)

\begin{tabular}{|c|c|c|c|c|c|c|c|c|c|c|}
\hline $\begin{array}{l}\text { Sample } \\
\text { Number }\end{array}$ & Plant ID & Location & Plant Species & Sample & Constituent & $\begin{array}{c}\text { Date } \\
\text { (Y/M/D) }\end{array}$ & Result & $\begin{array}{c}\text { Counting } \\
\text { Error }\end{array}$ & $\begin{array}{l}\text { Overall } \\
\text { Error }\end{array}$ & $\begin{array}{l}\text { Analysis } \\
\text { Size }(g)\end{array}$ \\
\hline B0BWP3 & B-8 & $\mathrm{K}-\mathrm{N}, 100$ Area & Balsamroot & Leaves & Co- 60 & 940505 & $0.00 \mathrm{E}+00$ & $4.32 \mathrm{E}-02$ & 4.32E-02 & 82 \\
\hline B0BWP3 & B-8 & $\mathrm{K}-\mathrm{N}, 100$ Area & Balsamroot & Leaves & Cs- 137 & 940505 & $4.04 \mathrm{E}-02$ & 4.15E-02 & 4.17E-02 & 82 \\
\hline B0BWP3 & B-8 & $\mathrm{K}-\mathrm{N}, 100$ Area & Balsamroot & Leaves & $\mathrm{K}-40$ & 940505 & $3.58 \mathrm{E}+01$ & $1.82 \mathrm{E}+00$ & $4.02 \mathrm{E}+00$ & 82 \\
\hline B0BWP3 & B-8 & $\mathrm{K}-\mathrm{N}, 100$ Area & Balsamroot & Leaves & Sr-90 & 940505 & 4.44E-02 & $9.49 \mathrm{E}-03$ & $1.26 \mathrm{E}-02$ & 82 \\
\hline BOBWP4 & B-8 & $\mathrm{K}-\mathrm{N}, 100$ Area & Balsamroot & Root & $\mathrm{Be}-7$ & 940505 & $2.78 \mathrm{E}-01$ & $1.39 \mathrm{E}-01$ & $1.42 \mathrm{E}-01$ & 302 \\
\hline B0BWP4 & B-8 & $\mathrm{K}-\mathrm{N}, 100$ Area & Balsamroot & Root & $\mathrm{Co}-60$ & 940505 & $4.05 \mathrm{E}-03$ & $1.07 \mathrm{E}-02$ & $1.07 \mathrm{E}-02$ & 302 \\
\hline B0BWP4 & B-8 & $\mathrm{K}-\mathrm{N}, 100$ Area & Balsamroot & Root & Cs-137 & 940505 & $8.70 \mathrm{E}-02$ & $1.62 \mathrm{E}-02$ & $1.84 \mathrm{E}-02$ & 302 \\
\hline B0BWP4 & B-8 & $\mathrm{K}-\mathrm{N}, 100$ Area & Balsamroot & Root & $\mathrm{K}-40$ & 940505 & $1.09 \mathrm{E}+01$ & $4.52 \mathrm{E}-01$ & $1.18 \mathrm{E}+00$ & 302 \\
\hline B0BWP4 & B-8 & $\mathrm{K}-\mathrm{N}, 100$ Area & Balsamroot & Root & Sr-90 & 940505 & $3.70 \mathrm{E}-02$ & $6.06 \mathrm{E}-03$ & $9.21 \mathrm{E}-03$ & 101 \\
\hline B0BWP5 & B-9 & K-N, 100 Area & Balsamroot & Leaves & $\mathrm{Be}-7$ & 940505 & $2.20 \mathrm{E}+00$ & $5.69 \mathrm{E}-01$ & $6.10 \mathrm{E}-01$ & 138 \\
\hline BOBWP5 & B-9 & $\mathrm{K}-\mathrm{N}, 100$ Area & Balsamroot & Leaves & $\mathrm{Co}-60$ & 940505 & $-5.15 \mathrm{E}-02$ & $3.45 \mathrm{E}-02$ & $3.49 \mathrm{E}-02$ & 138 \\
\hline B0BWP5 & B-9 & K-N, 100 Area & Balsamroot & Leaves & Cs-137 & 940505 & $-7.84 \mathrm{E}-03$ & $2.92 \mathrm{E}-02$ & $2.92 \mathrm{E}-02$ & 138 \\
\hline B0BWP5 & B-9 & K-N, 100 Area & Balsamroot & Leaves & $\mathrm{K}-40$ & 940505 & $3.68 \mathrm{E}+01$ & $1.57 \mathrm{E}+00$ & $4.00 \mathrm{E}+00$ & 138 \\
\hline B0BWP5 & B-9 & K-N, 100 Area & Balsamroot & Leaves & $\operatorname{Sr}-90$ & 940505 & $3.04 \mathrm{E}-02$ & $6.90 \mathrm{E}-03$ & $9.04 \mathrm{E}-03$ & 101 \\
\hline B0BWP6 & B-9 & $\mathrm{K}-\mathrm{N}, 100$ Area & Balsamroot & Root & $\mathrm{Be}-7$ & 940505 & $-4.34 \mathrm{E}-02$ & $6.63 \mathrm{E}-02$ & $6.65 \mathrm{E}-02$ & 373 \\
\hline B0BWP6 & B-9 & $\mathrm{K}-\mathrm{N}, 100$ Area & Balsamroot & Root & $\mathrm{Co}-60$ & 940505 & 4.41E-02 & $1.07 \mathrm{E}-02$ & $1.16 \mathrm{E}-02$ & 373 \\
\hline B0BWP6 & B-9 & K-N, 100 Area & Balsamroot & Root & Cs-137 & 940505 & $3.40 \mathrm{E}-02$ & $1.08 \mathrm{E}-02$ & $1.13 \mathrm{E}-02$ & 373 \\
\hline B0BWP6 & B-9 & $\mathrm{K}-\mathrm{N}, 100$ Area & Balsamroot & Root & $K-40$ & 940505 & $5.90 \mathrm{E}+00$ & $3.10 \mathrm{E}-01$ & $6.67 \mathrm{E}-01$ & 373 \\
\hline B0BWP6 & B-9 & $\mathrm{K}-\mathrm{N}, 100$ Area & Balsamroot & Root & Sr-90 & 940505 & $3.03 \mathrm{E}-02$ & $7.50 \mathrm{E}-03$ & $9.26 \mathrm{E}-03$ & 100 \\
\hline B0BWP7 & B-10 & $\mathrm{K}-\mathrm{N}, 100$ Area & Balsamroot & Root & $\mathrm{Be}-7$ & 940505 & $5.87 \mathrm{E}-03$ & $7.49 \mathrm{E}-02$ & $7.49 \mathrm{E}-02$ & 359 \\
\hline B0BWP7 & B-10 & K-N, 100 Area & Balsamroot & Root & $\mathrm{Co}-60$ & 940505 & $-2.78 \mathrm{E}-03$ & $8.99 \mathrm{E}-03$ & $8.99 \mathrm{E}-03$ & 359 \\
\hline B0BWP7 & B-10 & $\mathrm{K}-\mathrm{N}, 100$ Area & Balsamroot & Root & Cs- 137 & 940505 & $5.03 \mathrm{E}-02$ & $1.27 \mathrm{E}-02$ & $1.37 \mathrm{E}-02$ & 359 \\
\hline B0BWP7 & B-10 & K-N, 100 Area & Balsamroot & Root & $\mathrm{K}-40$ & 940505 & $7.46 \mathrm{E}+00$ & $3.45 \mathrm{E}-01$ & $8.22 \mathrm{E}-01$ & 359 \\
\hline B0BWP7 & B-10 & $\mathrm{K}-\mathrm{N}, 100$ Area & Balsamroot & Root & Sr -90 & 940505 & $3.17 \mathrm{E}-02$ & $7.12 \mathrm{E}-03$ & $9.10 \mathrm{E}-03$ & 100 \\
\hline B0BWP8 & B-10 & K-N, 100 Area & Balsamroot & Leaves & $\mathrm{Be}-7$ & 940505 & $2.64 \mathrm{E}+00$ & $5.72 \mathrm{E}-01$ & $6.30 \mathrm{E}-01$ & 120 \\
\hline B0BWP8 & B-10 & $\mathrm{K}-\mathrm{N}, 100$ Area & Balsamroot & Leaves & Co-60 & 940505 & $5.12 \mathrm{E}-03$ & $3.32 \mathrm{E}-02$ & $3.32 \mathrm{E}-02$ & 120 \\
\hline B0BWP8 & B-10 & K-N, 100 Area & Balsamroot & Leaves & Cs-137 & 940505 & $3.35 \mathrm{E}-02$ & $2.77 \mathrm{E}-02$ & $2.79 \mathrm{E}-02$ & 120 \\
\hline B0BWP8 & B-10 & K-N, 100 Area & Balsamroot & Leaves & $\mathrm{K}-40$ & 940505 & $2.83 \mathrm{E}+01$ & $1.54 \mathrm{E}+00$ & $3.22 \mathrm{E}+00$ & 120 \\
\hline B0BWP8 & B-10 & K-N, 100 Area & Balsamroot & Leaves & $\operatorname{Sr}-90$ & 940505 & $7.45 \mathrm{E}-03$ & $5.26 \mathrm{E}-03$ & $5.45 \mathrm{E}-03$ & 119 \\
\hline B0BWP9 & $P-4$ & K-N, 100 Area & Cymopterus & Leaves & $\mathrm{Be}-7$ & 940505 & $4.04 \mathrm{E}+00$ & $4.95 \mathrm{E}-01$ & $6.39 \mathrm{E}-01$ & 135 \\
\hline В0ВWP9 & $P-4$ & K-N, 100 Area & Cymopterus & Leaves & Co-60 & 940505 & $1.20 \mathrm{E}-02$ & $3.68 \mathrm{E}-02$ & $3.68 \mathrm{E}-02$ & 135 \\
\hline B0BWP9 & P-4 & $\mathrm{K}-\mathrm{N}, 100$ Area & Cymopterus & Leaves & Cs- 137 & 940505 & $-3.72 E-03$ & $3.52 \mathrm{E}-02$ & $3.52 \mathrm{E}-02$ & 135 \\
\hline B0BWP9 & P-4 & K-N, 100 Area & Cymopterus & Leaves & $\mathrm{K}-40$ & 940505 & $3.28 \mathrm{E}+01$ & $\cdot 1.53 \mathrm{E}+00$ & $3.62 \mathrm{E}+00$ & 135 \\
\hline B0BWP9 & P-4 & $\mathrm{K}-\mathrm{N}, 100$ Area & Cymopterus & Leaves & Sr -90 & 940505 & $7.37 \mathrm{E}-03$ & $6.01 \mathrm{E}-03$ & $6.19 \mathrm{E}-03$ & 99 \\
\hline B0BWQ0 & B-11 & N-D, 100 Area & Balsamroot & Leaves & $\mathrm{Be}-7$ & 940505 & $3.67 \mathrm{E}+00$ & $6.97 \mathrm{E}-01$ & $7.88 \mathrm{E}-01$ & 86 \\
\hline B0BWQ0 & B-11 & N-D, 100 Area & Balsamroot & Leaves & $\mathrm{Co}-60$ & 940505 & $-3.75 \mathrm{E}-03$ & $5.98 \mathrm{E}-02$ & $5.98 \mathrm{E}-02$ & 86 \\
\hline B0BWQ0 & B-11 & N-D, 100 Area & Balsamroot & Leaves & Cs- 137 & 940505 & $4.68 \mathrm{E}-02$ & $4.67 \mathrm{E}-02$ & $4.69 \mathrm{E}-02$ & 86 \\
\hline B0BWQ0 & B-i1 & N-D, 100 Area & Balsamroot & Leaves & $\mathrm{K}-40$ & 940505 & $3.99 \mathrm{E}+01$ & $2.11 \mathrm{E}+00$ & $4.51 \mathrm{E}+00$ & 86 \\
\hline B0BWQ0 & B-11 & N-D, 100 Area & Balsamroot & Leaves & $\mathrm{Sr}-90$ & 940505 & $2.17 \mathrm{E}-02$ & $5.12 \mathrm{E}-03$ & $6.66 \mathrm{E}-03$ & 86 \\
\hline B0BWQ1 & B-11 & N-D, 100 Area & Balsamroot & Root & $\mathrm{Be}-7$ & 940505 & $1.26 \mathrm{E}-01$ & $1.42 \mathrm{E}-01$ & $1.43 \mathrm{E}-01$ & 163 \\
\hline B0BWQ1 & B-11 & N-D, 100 Area & Balsamroot & Root & $\mathrm{Co}-60$ & 940505 & $2.69 \mathrm{E}-02$ & $1.58 \mathrm{E}-02$ & $1.60 \mathrm{E}-02$ & 163 \\
\hline B0BWQ1 & B-11 & N-D, 100 Area & Balsamroot & Root & Cs- 137 & 940505 & $7.55 \mathrm{E}-02$ & $1.98 \mathrm{E}-02$ & $2.12 \mathrm{E}-02$ & 163 \\
\hline B0BWQ1 & B-11 & N-D, 100 Area & Balsamroot & Root & $\mathrm{K}-40$ & 940505 & $7.48 \mathrm{E}+00$ & $5.38 \mathrm{E}-01$ & $9.22 \mathrm{E}-01$ & 163 \\
\hline
\end{tabular}


Table B.1. (con't)

\begin{tabular}{|c|c|c|c|c|c|c|c|c|c|c|}
\hline $\begin{array}{l}\text { Sample } \\
\text { Number }\end{array}$ & Plant ID & Location & Plant Species & Sample & Constituent & $\begin{array}{c}\text { Date } \\
(\mathrm{Y} / \mathrm{M} / \mathrm{D})\end{array}$ & Result & $\begin{array}{c}\text { Counting } \\
\text { Error }\end{array}$ & $\begin{array}{c}\text { Overall } \\
\text { Error }\end{array}$ & $\begin{array}{c}\text { Analysis } \\
\text { Size (g) }\end{array}$ \\
\hline B0BWQ1 & B-11 & N-D, 100 Area & Balsamroot & Root & Sr -90 & $940505^{\circ}$ & $5.78 \mathrm{E}-02$ & $8.20 \mathrm{E}-03$ & $1.37 \mathrm{E}-02$ & 106 \\
\hline B0BWQ2 & B-12 & N-D, 100 Area & Balsamroot & Leaves & $\mathrm{Be}-7$ & 940505 & $1.66 \mathrm{E}+00$ & $2.01 \mathrm{E}-01$ & 2.60E-01 & 172 \\
\hline BOBWQ2 & B-12 & N-D, 100 Area & Balsamroot & Leaves & Co-60 & 940505 & $3.43 \mathrm{E}-03$ & $1.29 \mathrm{E}-02$ & $1.29 \mathrm{E}-02$ & 172 \\
\hline BOBWQ2 & B-12 & N-D, 100 Area & Balsamroot & Leaves & Cs- 137 & 940505 & $2.15 \mathrm{E}-03$ & $9.62 \mathrm{E}-03$ & $9.62 \mathrm{E}-03$ & 172 \\
\hline B0BWQ2 & B-12 & N-D, 100 Area & Balsamroot & Leaves & $\mathrm{K}-40$ & 940505 & $3.38 \mathrm{E}+01$ & $6.92 \mathrm{E}-01$ & $3.45 E+00$ & 172 \\
\hline B0BWQ2 & B-12 & N-D, 100 Area & Balsamroot & Leaves & Sr -90 & 940505 & $6.85 \mathrm{E}-03$ & $5.53 \mathrm{E}-03$ & $5.70 \mathrm{E}-03$ & 112 \\
\hline B0BWQ3 & B-12 & N-D, 100 Area & Balsamroot & Root & $\mathrm{Be}-7$ & 940505 & $2.84 \mathrm{E}-02$ & $1.01 \mathrm{E}-01$ & $1.01 \mathrm{E}-01$ & 215 \\
\hline B0BWQ3 & B-12 & N-D, 100 Area & Balsamroot & Root & $\mathrm{Co}-60$ & 940505 & $0.00 \mathrm{E}+00$ & $1.00 \mathrm{E}-02$ & $1.00 \mathrm{E}-02$ & 215 \\
\hline B0BWQ3 & B-12 & N-D, 100 Area & Balsamroot & Root & Cs-137 & 940505 & $4.61 \mathrm{E}-02$ & $1.74 \mathrm{E}-02$ & $1.80 \mathrm{E}-02$ & 215 \\
\hline B0BWQ3 & B-12 & N-D, 100 Area & Balsamroot & Root & $\mathbf{K}-40$ & 940505 & $6.94 \mathrm{E}+00$ & 4.45E-01 & 8.25E-01 & 215 \\
\hline B0BWQ3 & B-12 & N-D, 100 Area & Balsamroot & Root & Sr -90 & 940505 & $2.68 \mathrm{E}-02$ & $4.20 \mathrm{E}-03$ & $6.53 \mathrm{E}-03$ & 102 \\
\hline B0BWQ4 & B-13 & D-H, 100 Area & Balsamroot & Leaves & $\mathrm{Be}-7$ & 940505 & $1.55 \mathrm{E}+00$ & $2.47 \mathrm{E}-01$ & $2.91 \mathrm{E}-01$ & 203 \\
\hline B0BWQ4 & B-13 & D-H, 100 Area & Balsamroot & Leaves & $\mathrm{Co}-60$ & 940505 & $9.64 \mathrm{E}-04$ & $1.53 \mathrm{E}-02$ & $1.53 \mathrm{E}-02$ & 203 \\
\hline B0BWQ4 & B-13 & D-H, 100 Area & Balsamroot & Leaves & Cs- 137 & 940505 & $1.14 \mathrm{E}-02$ & $1.22 \mathrm{E}-02$ & $1.23 \mathrm{E}-02$ & 203 \\
\hline B0BWQ4 & B-13 & D-H, 100 Area & Balsamroot & Leaves & $K-40$ & 940505 & $3.07 \mathrm{E}+01$ & $8.33 \mathrm{E}-01$ & $3.18 \mathrm{E}+00$ & 203 \\
\hline B0BWQ4 & B-13 & D-H, 100 Area & Balsamroot & Leaves & Sr-90 & 940505 & $2.93 \mathrm{E}-02$ & $6.27 \mathrm{E}-03$ & $8.19 \mathrm{E}-03$ & 100 \\
\hline B0BWQ5 & B-13 & D-H, 100 Area & Balsamroot & Root & $\mathrm{Be}-7$ & 940505 & $2.29 \mathrm{E}-01$ & $1.26 \mathrm{E}-01$ & $1.28 \mathrm{E}-01$ & 193 \\
\hline B0BWQ5 & B-13 & D-H, 100 Area & Balsamroot & Root & $\mathrm{Co}-60$ & 940505 & $-9.34 \mathrm{E}-03$ & $1.20 \mathrm{E}-02$ & $1.21 \mathrm{E}-02$ & 193 \\
\hline B0BWQ5 & B-13 & D-H, 100 Area & Balsamroot & Root & Cs- 137 & 940505 & $6.00 \mathrm{E}-02$ & $1.93 \mathrm{E}-02$ & $2.02 \mathrm{E}-02$ & 193 \\
\hline B0BWQ5 & B-13 & D-H, 100 Area & Balsamroot & Root & $\mathrm{K}-40$ & 940505 & $7.05 \mathrm{E}+00$ & $5.14 \mathrm{E}-01$ & $8.72 \mathrm{E}-01$ & 193 \\
\hline B0BWQ5 & B-13 & D-H, 100 Area & Balsamroot & Root & $\mathrm{Sr}-90$ & 940505 & 8.47E-02 & $7.23 \mathrm{E}-03$ & $1.65 \mathrm{E}-02$ & 101 \\
\hline B0BWQ6 & B-14 & F, 100 Area & Balsamroot & Leaves & $\mathrm{Be}-7$ & 940505 & $1.37 \mathrm{E}+00$ & $2.55 \mathrm{E}-01$ & $2.89 \mathrm{E}-01$ & 160 \\
\hline B0BWQ6 & B-14 & $\mathrm{F}, 100$ Area & Balsamroot & Leaves & $\mathrm{Co}-60$ & 940505 & $4.38 \mathrm{E}-02$ & $1.63 \mathrm{E}-02$ & $1.69 \mathrm{E}-02$ & 160 \\
\hline B0BWQ6 & B-14 & F, 100 Area & Balsamroot & Leaves & Cs- 137 & 940505 & $8.54 \mathrm{E}-03$ & $1.48 \mathrm{E}-02$ & $1.49 \mathrm{E}-02$ & 160 \\
\hline B0BWQ6 & B-14 & F, 100 Area & Balsamroot & Leaves & $\mathrm{K}-40$ & 940505 & $3.48 \mathrm{E}+01$ & $1.05 E+00$ & $3.63 E+00$ & 160 \\
\hline B0BWQ6 & B-14 & F, 100 Area & Balsamroot & Leaves & Sr-90 & 940505 & $1.24 \mathrm{E}-02$ & $4.14 \mathrm{E}-03$ & $4.80 \mathrm{E}-03$ & 103 \\
\hline B0BWQ7 & B-14 & F, 100 Area & Balsamroot & Root & $\mathrm{Be}-7$ & 940505 & $-1.44 \mathrm{E}-04$ & $8.62 \mathrm{E}-04$ & 8.63E-04 & 212 \\
\hline B0BWQ7 & B-14 & F, 100 Area & Balsamroot & Root & $\mathrm{Co}-60$ & 940505 & $-2.64 \mathrm{E}-03$ & $9.39 \mathrm{E}-03$ & $9.40 \mathrm{E}-03$ & 212 \\
\hline B0BWQ7 & B-14 & F, 100 Area & Balsamroot & Root & Cs-137 & 940505 & $3.55 \mathrm{E}-02$ & $1.70 \mathrm{E}-02$ & $1.74 \mathrm{E}-02$ & 212 \\
\hline B0BWQ7 & B-14 & $\mathrm{F}, 100$ Area & Balsamroot & Root & $K-40$ & 940505 & $6.46 \mathrm{E}+00$ & $4.41 \mathrm{E}-01$ & $7.83 \mathrm{E}-01$ & 212 \\
\hline B0BWQ7 & B-14 & F, 100 Area & Balsamroot & Root & Sr-90 & 940505 & $9.70 \mathrm{E}-03$ & $3.40 \mathrm{E}-03$ & $3.90 \mathrm{E}-03$ & 101 \\
\hline
\end{tabular}




\section{Distribution}

No. of

Copies

\section{OFFSITE}

2 DOE/Office of Scientific and Technical Information

2 Golder Associates

4104 148th Avenue N.E.

Redmond, WA 98052

Attn: L. Swenson

Attn: W. Wright

J. Yoke1

Washington State Department of Ecology

M. S. PV-11

Olympia, WA 98504-8711

2 Environmental Restoration/

Waste Mangement

Yakama Indian Nation

P.O. Box 151

Toppenish, WA 98948

Attn: R. Jim

Attn: M. Bauer

5 Confederated Tribes of the Umatilla Indian Reservation

P.O. Box 638

Pendleton, OR 97801

Attn: J. Ebaugh

Attn: J. R. Wilkinson

Attn: A. Childs

Attn: R. George

Attn: K. Robson

2 Nez Perce Tribe

P.O. Box 365

Lapwai, ID 83540-0365

Attn: R. Cruz

Attn: D. Powaukee
No. of

Copies

3 Washington State Department of Health

Division of Radiation Protection

Airdustrial Center

Building 5, M.S. C-13

Olympia, WA 98503

Attn: J. Erickson

Attn: L. Albin

Attn: D. Thatcher

\section{ONSITE}

10 DOE Richland Operations Office
R. F. Brich
J. B. Hall (5)
H4-83
R. D. Hildebrand
A5-55
P. S. Kube
A5-55
D. C. Ward
A5-15
B. A. Ward
A5-55
H4-83

2 U.S. Environmental Protection Agency
L. E. Gadbois
B5-01
P. S. Innes
B5-01

7 Westinghouse Hanford Company

L. P. Diediker

J. J. Dorian

$\mathrm{T} 1-30$

E. M. Greager

H6-20

A. R. Johnson

H6-20

C. J. Perkins

H6-20

J. W. Schmidt

$\mathrm{X} 0-21$

Public Reading Room

$\mathrm{H} 2-53$

4 Bechtel Hanford, Inc.

G. E. Fitzgibbon H6-07

K. A. Gano X0-21

K. M. Leonard H6-20

S. G. Weiss

H4-90 
No. of

Conies

52 Pacific Northwest Laboratory
E. J. Antonio
M. L. Blanton
C. A. Brandt
R. W. Bryce
N. A. Cadoret
L. L. Cadwell
A. T. Cooper
D. D. Dauble
R. L. Dirkes
S. L. Friant
D. R. Geist
R. W. Hanf, Jr.
D. W. Harvey
R. E. Jaquish (3)
E. W. Lusty
P. R. Nickens
G. W. Patton

K6-75

$\mathrm{K} 6-75$

K6-84

K6-96

K6-75

$\mathrm{K} 6-84$

$\mathrm{K} 6-75$

K6-54

K6-75

$\mathrm{K} 6-77$

$\mathrm{K} 6-54$

K6-75

K6-75

$\mathrm{K} 6-75$

K6-79

K6-75

K6-75
No. of

Conies

T. M. Poston (20) K6-75

J. K. Soldat

M. E. Thiede

B. L. Tiller

M. K. Wright

Publishing Coordination

SESP Historical Files/

R. L. Dirkes (2)

Technical Report Files (5)

Routing

R. W. Bryce

K9-34

R. M. Ecker

SE-UI

M. J. Graham

S. A. Rawson

R. L. Skaggs

P. C. Hays - last

K9-38

K9-34

K9-34

K9-41 